\title{
EVALUATION OF ANTIOXIDANT AND ANTIBACTERIAL ACTIVITIES OF FRESH AND FREEZE-DRIED SELECTED FRUIT JUICES
}

\author{
NARITSARA TOOBPENG ${ }^{1}$, PANNAPA POWTHONG ${ }^{2}$, PATTRA SUNTORNTHITICHAROEN ${ }^{3 *}$ \\ ${ }^{1}$ Biomedical Sciences Graduate Program, Department of Medical Sciences, Faculty of Science, Rangsit University, Pathum Thani 12000, \\ Thailand. ${ }^{2}$ Faculty of Medical Technology, Rangsit University, Pathum Thani 12000, Thailand. ${ }^{3}$ Department of Medical Sciences, Faculty of \\ Science, Rangsit University, Pathum Thani 12000, Thailand. Email: pattra.s@rsu.ac.th
}

Received: 12 April 2017, Revised and Accepted: 24 May 2017

ABSTRACT

Objective: The objective of this investigation was to assess the biological activity of fresh fruit juices and freeze dried fruit juices including antioxidant and antibacterial activity.

Methods: The fruits used in this study were Schleichera oleosa (Lour.) Oken, Carissa carandas L., and Sandoricum koetjape (Burm.f.) Merr. The fresh juice sample (FJS) was extracted and also prepared for freeze drying sample (FDS) by freeze dryer. The antioxidant capacity of FJS and FDS was assessed using the 2,2-diphenyl-1-picryl-hydrazyl (DPPH) assays and determination for total phenolic contents (TPCs) by Folin-Ciocalteu's reagent. Antimicrobial tests were carried out by agar diffusion methods and evaluated by measuring the zone of inhibition against 10 isolates of pathogenic bacteria.

Results: The highest antioxidant activity of FJS and FDS was found in S. oleosa and related to the scavenging effect on DPPH radical of FJS (100\% concentration) and FDS (200 mg/ml) was found in the value of $93.05 \pm 0.31 \%$ and $82.18 \pm 0.33 \%$, respectively. TPC of FJS and FDS from $S$. oleosa at the same concentration as in the DPPH assay was 1,003.53 $\pm 1.96 \mathrm{ug}$ GAE/300 g of matter and $827.77 \pm 23.15 \mathrm{ug}$ GAE/100 ml of matter, respectively. Both FJS and FDS revealed antibacterial activity. The zone of inhibition of FJS was ranging from $8.3 \pm 0.6$ to $24.0 \pm 1.0$ mm and FDS values of 7.3 $\pm 0.5-21.0 \pm 0.9 \mathrm{~mm}$. The most potent sample against bacteria was FJS and FDS from S. oleosa which FJSs against Escherichia coli ESBL and FDS against Pseudomonas aeruginosa MDR2.

Conclusion: Fruit juices of $S$. oleosa, C. carandas, and $S$. koetjape associated with antioxidant activity in the form of both fresh fruit juices and FDS. The result of this study showed that the value of DPPH and TPCs were slightly decreased in FDS compare with fresh juice. Screening of FJS and FDS of C. carandas revealed broad spectrum antibacterial activity. However, FJS of S. oleosa and S. koetjape showed inhibition of growth of bacteria, but few of FDS from these fruits could inhibit limited bacterial isolates. For antibacterial activity against pathogenic bacteria, the samples of fresh and freeze dried exhibited a different degree of activity.

Keywords: Fresh juices, Freeze dried, Antibacterial, Antioxidant, Fruit.

(C) 2017 The Authors. Published by Innovare Academic Sciences Pvt Ltd. This is an open access article under the CC BY license (http://creativecommons. org/licenses/by/4. 0/) DOI ttp://dx.doi.org/10.22159/ajpcr.2017.v10i9.19099

\section{INTRODUCTION}

In the human living system, oxidation is known to involve a series of free radical in the form of reactive oxygen and nitrogen species-mediated chain reactions which antioxidants protect the body from damage caused by harmful molecules. Antioxidant could reduce reactive oxygen species or delay the chain reaction of an oxidizable substance, and scavenge free radical. Phenolic compounds are phytochemicals which play a major role in the protection of oxidation processes. There is some comfort knowing that such antioxidants are purified from natural products that have been consumed for generations [1]. Thus, they have a wide range of biological activities, such as excellent antioxidant activities and protected against antimicrobial activities. The researcher reported that the amount of phenolic compounds in plant depends on cultivation techniques, cultivar, growing conditions, extraction methods, extraction temperature, and ripening process, as well as processing and storage conditions [2].

Apart from plants, some naturally consumed fruits also have important bioactivity properties. Many of the health advantages of fruits are attributed to the phytonutrients or bioactive compounds within plants that provide health benefits beyond that of the traditional nutrients. Among many advantageous properties, phytonutrients contribute direct and indirect bioactivity attributable to their free radical scavenging ability [3]. The majority of other compounds may be vitamin C, vitamin $\mathrm{E}, \beta$-carotene, or flavonoids, which there are strong antioxidant activities [4]. Fruit juices are primarily considered as a main source of phenolic compounds. The high in polyphenols contents caused in a high antioxidant activity [5]. Natural products have interesting biological activity. It possesses an interesting challenge in organic chemistry, and has remarkable structural diversity and biological characteristics, providing researchers with exciting possibilities to develop novel molecular entities for human therapeutics [6]. Many natural products have a variety of biological activities and have always been a significant source of new lead compounds in pharmaceutical industries [7]. Increasing antibiotic resistant microbe problems lead to urgent needs for new antimicrobial agents, especially new sources of the agents.

Thailand is a major source of tropical plant varieties, which are associated with many medicinal properties. Fruits are the excellent sources of antioxidant and antimicrobial from natures. There are a large number of fruits in Thailand. Schleichera oleosa (Lour.) Oken. is classified in a family of Sapindaceae. The fruits tasted sour and could prepare for fruit juices. Many parts of $S$. oleosa have been studied about pharmacological importance, including antioxidant, antiulcer, anticancer, and antimicrobial activities [8]. Carissa carandas L. belongs to family Apocynaceae. The crude extracts of $C$. carandas leaves have 
been evaluated for antioxidant [9]. In addition, fruits of $C$. carandas were examined for antioxidant and nutraceutical property [10]. In addition, fruits, leaf, and root of $C$. carandas has been reported from the crude extract and their different fractions and isolates which were evaluated for antioxidant [9]. Sandoricum koetjape (Burm.f.) Merr. is the plant belonging to the family Meliaceae. The phytochemical and pharmacological properties from fruits, seeds, leaves, and bark were studied [11]. Numerous triterpenoids have been found from stem bark. Although earlier reports show the antimicrobial and antioxidant potential in some part of them, but there are many of them that remain unexplored.

In the season of fruits, lots of product is easily found in the market. The limitation of the short season of fresh fruit and large amounts of product in the market could be solved by processing the fruits. Freeze dried method is the way that decrease water from the substances [12]. This method is a more powerful process for preserving the contents of the fruits for value adding of fruits when its flood in the market. The composition of the phenolic compounds in juice products depends on the juice treating application [13]. There are physical-chemical changes in fruit products due to lyophilize processing. Values of antioxidant activity also depend strongly on the preparation of samples. The stability of components and bioactive properties require exploring for proving the properties of processing fruits while the fruits are limited in very short periods of the year. The processed food in the form of freeze drying could be used for promoting for healthy food and more precious than fresh fruits which are easily denatured.

This study aims to evaluate the antioxidant and antimicrobial activities of fresh fruit juices and freeze dried fruit juices from S. oleosa, C. carandas, and S. koetjape.

\section{METHODS}

\section{Bacterial strains}

About 10 isolates of multidrug-resistant bacteria, which contains seven Gram-negative strains: Pseudomonas aeruginosa MDR1, P. aeruginosa MDR2, Klebsiella pneumoniae ESBL, Escherichia coli P174 ESBL, E. coli ESBL, Acinetobacter baumannii MDR1, and A. baumannii MDR2 and three Gram-positive strains: Enterococcus faecalis, Staphylococcus aureus methicillin-resistant $S$. aureus MRSA1, and S. aureus MRSA2 were used. Bacteria were stored at $-80^{\circ} \mathrm{C}$.

\section{Chemicals}

2, 2-diphenyl-1-picryl-hydrazyl (DPPH), Gallic acid, and Folin-Ciocalteu reagents were purchased from Sigma-Aldrich, Germany. Ascorbic acid was purchased from Unilab, England. All other chemicals and reagents used in the study were of analytical grade.

\section{Collection of plant material}

Three ripe fruits, S. oleosa (Lour.) Oken, C. carandas L., and S. koetjape (Burm.f.) Merr., were obtained from the Nakhon Ratchasima province, Thailand on July 2016. The fruits were washed with tap water and air dried before used.

\section{Preparation of fresh fruit juice samples}

About $300 \mathrm{~g}$ of each ripe fruit were washed, peeled and cut into smaller bits, then was extracted by extractor for 30 seconds. The fruit juice was filtered through a Whatman filter paper and kept as fresh juice samples (FJS) at $-20^{\circ} \mathrm{C}$ until use.

\section{Preparation of freeze dried samples (FDS)}

About $100 \mathrm{ml}$ of fresh fruit juice has been freeze dried by vacuum freeze dryer for $24 \mathrm{hrs}$. Then, the FDS were kept on $-20^{\circ} \mathrm{C}$ until use.

\section{Antioxidant activity}

DPPH assay

The potential antioxidant activities of the samples were determined using DPPH according to Powthong and co-workers (2013) [14]. Briefly,
$100 \mu \mathrm{l}$ of the fresh juice $(100 \%, 50 \%, 25 \%, 12.5 \%$, and $6.25 \%$ serially) and FDS (concentration of 12.5, 25, 50,100, and $200 \mathrm{mg} / \mathrm{ml}$ ) prepared in ethanol was mixed with $100 \mu$ l of $0.2 \mathrm{mM} \mathrm{DPPH}$ (DPPH, Sigma) prepared in methanol. The mixture was shaken vigorously and incubated under dark at room temperature for 30 minutes, and the absorbance was then measured at $517 \mathrm{~nm}$ against a blank. Simultaneously, a control was prepared without sample extracts. The capability to scavenge the DPPH radical was calculated using the following equation:

DPPH scavenging effect $(\%)=\left[\left(A_{0}-A_{1} / A_{0}\right) \times 100\right]$

Where $A_{0}$ was the absorbance of the control reaction and $A_{1}$ was the absorbance in the presence of the sample. All measurements were performed in triplicate.

\section{Determination of total phenol content}

Total phenolic compounds were determined using Folin-Ciocalteu's method [14]. Briefly, $20 \mu \mathrm{l}$ of the fresh juice $(100 \%, 50 \%, 25 \%$, $12.5 \%$, and $6.25 \%$ serially) and FDS (concentration of $12.5,25,50$, 100 , and $200 \mathrm{mg} / \mathrm{ml}$ ) prepared in ethanol was mixed with $100 \mu \mathrm{l}$ of Folin-Ciocalteu's reagent. Then, $80 \mu \mathrm{l}$ of $2 \%$ aqueous sodium carbonate was added into the mixture. The mixture was incubated for 30 minutes, and the absorbance of the mixture was measured at $765 \mathrm{~nm}$ against the reagent blank. The content of total phenol was calculated on the basis of the calibration curve of gallic acid, and the results were expressed as $\mu \mathrm{g}$ of gallic acid equivalents (GAEs) per $300 \mathrm{~g}$ or $100 \mathrm{ml}$ of matter. All measurements were performed in triplicate.

\section{Screening of antibacterial activity by agar diffusion method}

Antimicrobial activity was determined by agar diffusion methods. All microorganisms were first left $16-18 \mathrm{hrs}$ at $37^{\circ} \mathrm{C}$ in nutrient agar base, then touch at least colonies with a sterile loop for inoculum into $0.85 \%$ $\mathrm{NaCl}$. Cellular density of inoculum was obtained by optical densities measured at $625 \mathrm{~nm}$ by a spectrophotometer to obtain the required bacterial concentration as $10^{8} \mathrm{CFU} / \mathrm{ml}$ or OD of $0.08-0.1$ [15].

A suspension of any tested bacteria containing about $10^{8} \mathrm{CFU} / \mathrm{ml}$ was spread on a Mueller-Hinton agar plate by three-way swab technique using sterile cotton swabs. For FJS, the Mueller-Hinton agar plate was pouched by cork borer $(\varnothing 6 \mathrm{~mm})$, and $30 \mu \mathrm{l}$ of the fresh samples were added into agar well. Gentamicin $(300 \mu \mathrm{g})$ was used as positive control.

FDS were prepared in DMSO at a concentration of $200 \mathrm{mg} / \mathrm{ml}$. Paper discs were placed on Mueller-Hinton agar plates containing tested bacteria then added $10 \mu \mathrm{l}$ of each FDS into paper disk. Which each paper disc contains $2 \mathrm{mg}$ of FDS. $10 \mu \mathrm{l}$ of DMSO were tested as a negative control. The plates were then incubated at $37^{\circ} \mathrm{C}$ for $24 \mathrm{hrs}$, and the diameter of the inhibition zone was measured. Triplicates of each sample have been done. The results were revealed by the average \pm standard deviation (SD).

\section{RESULTS AND DISCUSSION}

The difference in total antioxidant capacity (DPPH) and total phenolic content (TPC) among selected fresh fruit juice and its FDS showed in Figs. 1 and 2. DPPH scavenging activity was varied in all selected fruit juice, and its freeze-dried form. It was exhibited that the DPPH scavenging activity of FJSs was ranging from $4.50 \pm 3.50 \%$ to $93.05 \pm 0.31 \%$. Among the FJSs, the highest scavenging activity was found in FJSs of S. oleosa with a value of $93.05 \pm 0.31 \%$ at a concentration of $100 \%$. While the DPPH scavenging activity of FDS was ranging from $5.67 \pm 2.60 \%$ to $82.18 \pm 0.33 \%$. Among the FDS, the highest scavenging activity was found in S. oleosa with a value of $82.18 \pm 0.33 \%$ at a concentration of $200 \mathrm{mg} / \mathrm{ml}$. We found that when various concentrations of either fresh fruit juice or its FDS were subjected to determined total antioxidant capacity, DPPH levels revealed increasing in a dose-dependent manner. Moreover, DPPH radical scavenging activity values of FDS showed a nonstatistically significant decreased when compared to its fresh fruit juice. 


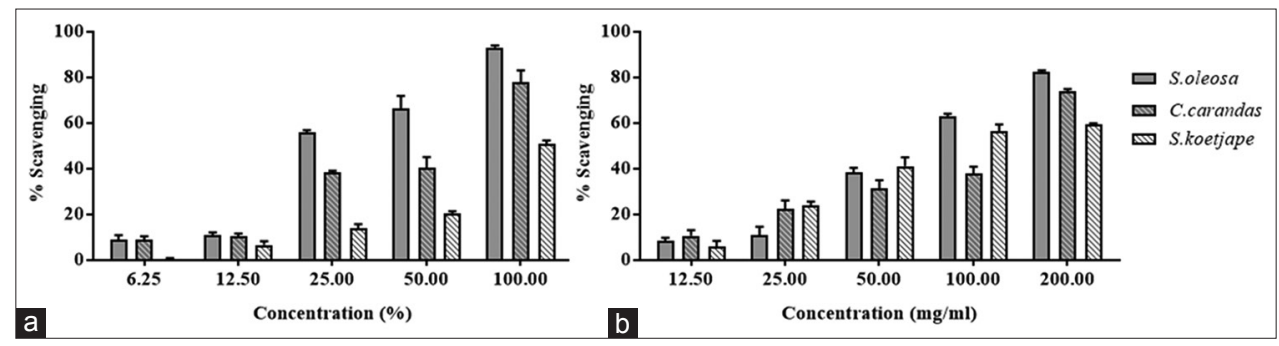

Fig. 1: The free radical scavenging activity of fruit juice samples and freeze dried samples. Antioxidant activity is expressed as the mean \pm standard deviation. (a) The free radical scavenging activity of fresh juice samples. (b) The free radical scavenging activity of freeze-dried samples

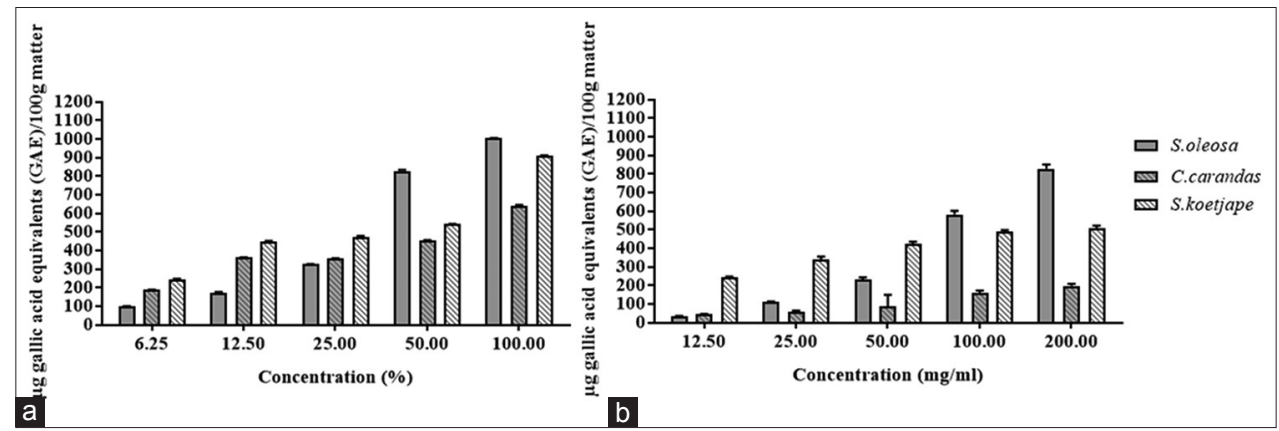

Fig. 2: Total phenolic contents of fruit juice samples and freeze dried samples. Antioxidant activity is expressed as the mean \pm standard deviation. (a) Total phenolic contents of fresh juice samples. (b) Total phenolic contents of freeze-dried samples

In addition, the TPC of FJSs ranging from $100.30 \pm 0.52 \mathrm{mg} \mathrm{GAE} / 300 \mathrm{~g}$ of matter to $1,003.53 \pm 1.96 \mathrm{mg} \mathrm{GAE} / 300 \mathrm{~g}$ of matter. Among the FJSs, the highest TPC was found in FJSs of $S$. oleosa with a value of $1,003.53 \pm 1.96 \mathrm{mg} \mathrm{GAE} / 300 \mathrm{~g}$ of matter at a concentration of $100 \%$. The TPC of FDS was ranging from $32.00 \pm 4.38 \mathrm{mg} \mathrm{GAE} / 100 \mathrm{ml}$ of matter to $827.77 \pm 23.15 \mathrm{mg} \mathrm{GAE} / 100 \mathrm{ml}$ of matter. Furthermore, a highest TPC was found in FDS of $S$. oleosa with a value of $827.77 \pm 23.15 \mathrm{mg}$ $\mathrm{GAE} / 100 \mathrm{ml}$ of matter. It was observed that the concentration of TPC, both fresh fruit juice and its FDS increased in a dose-dependent manner. TPC values of FDS showed a nonstatistically significant decreased when compared to its fresh fruit juice same as we observed in DPPH assay.

The data presented in the study demonstrated that $S$. oleosa has phenolic content and show excellent activity against DPPH radicals. Previous phytochemical studies of $S$. oleosa fruit showed that it contains terpenoids, flavonoids, tannins, and steroids [16]. From this result suggests that $S$. oleosa is a good choice for medical used or cosmetic agent against free-radical-associated oxidative damage [17]. Therefore, it can be further subjected to isolate the therapeutic antioxidant compounds activity and evaluate their pharmacologically.

In a general way, the antioxidant values increased with the increase of concentration, possibly due to the higher extraction of antioxidant phenolic compounds. This fact can be associated with higher concentration of fresh juice and FDS. Moreover, we observed nonsignificant differences in both total antioxidant capacity (DPPH) and TPC between fresh fruit juice and its FDS. It was revealed that the value of DPPH and TPC was slightly decreased according to extraction procedure. In addition, there is some previous research which describes the extraction process depended on antioxidant activity of fresh juice from other plants. Banana peels were revealed their antioxidant capacities of various extracts using DPPH assays [18]. Phytochemical properties of fruit extract of Elaeocarpus ganitrus were also determined for phenolic and flavonoid compounds [19]. Yan and Kerr (2013) reported that higher temperature used during vacuum belt drying of pomace negatively impacted the TPC [20]. The results of this study are also similar to those reported by Sogi et al. (2013) for mango peel and kernel drying, they used four different drying methods; freeze drying $-20^{\circ} \mathrm{C}$, hot air drying at $60^{\circ} \mathrm{C}$, vacuum drying at $60^{\circ} \mathrm{C}$, and infra-red. The highest value of total phenolic was noticed in FDS and hot air drying at $60^{\circ} \mathrm{C}$ was lower than FDS [21]. According to Chellaram et al., 2014 [22] purposed that the preparation of sample (freeze dehydration or lyophilization) is the cause of decreasing in antioxidant activity. The main reason may be the loss of phenolic compounds, which are the heat-sensitive bioactive compounds that can indicate the quantity of antioxidant activity. Therefore, the results of our study are similar to those in the previous reports and indicate that extraction process may serve as a slightly decreasing in antioxidant activity.

Identification of the antioxidants in S. oleosa, which are responsible for hydrogen and electron donation and metal chelation will supplement the findings of the study. The result of the trial is a good way to develop utilizing S. oleosa extracts as a commercial antioxidant will be greatly advanced through optimization of the extraction procedure. In another hand, it can also be further developed as a product to increase the value of the $S$. oleosa on the season, which has a large amount of output.

The result of in vitro antimicrobial activity of fresh and FDS was shown in Table 1 with different samples against the microorganisms. DMSO did not inhibit the growth of testing microorganisms (unpublished data), while gentamicin did. The fresh juice samples, S. oleosa $\mathrm{pH} 4.24$, C. carandas $\mathrm{pH} 3.41$, and $S$. koetjape $\mathrm{pH} 4.31$, showed a zone of inhibition against almost all of the tested pathogenic bacteria with a zone of inhibition ranging from $8.3 \pm 0.6$ to $24.0 \pm 1.0 \mathrm{~mm}$. Among FJS, the most effective samples were FJS of $S$. oleosa against $E$. coli ESBL. For the FDS ( $2 \mathrm{mg}$ ), a zone of inhibition was ranging from $7.3 \pm 0.5$ to $21.0 \pm 0.9 \mathrm{~mm}$. The highest zone was FDS from $S$. oleosa. Only C. carandas inhibited all kinds of the tested bacteria $(7.7 \pm 0.5$ to $17.0 \pm 1.2 \mathrm{~mm})$ while $S$. oleosa and S. koetjape were observed against P. aeruginosa MDR2. But S. oleosa could inhibit one more bacteria, $S$. aureus MRSA1.

In this study, antibacterial activity of fresh juices of all three fruits showed a broad spectrum against pathogenic bacteria. In general, $\mathrm{pH}$ of three selected fresh fruit juice samples was lower than 5 . Evaluation of $\mathrm{pH}$ in foods is important because bacteria require a neutral $\mathrm{pH}$ environment to grow. The low $\mathrm{pH}$ of these fruit juices may affect the growth of bacteria. Previous study, leaf and seed extract of $S$. oleosa showed antibacterial 
Table 1: Antibacterial activity of FJS and FDS on different bacterial strains

\begin{tabular}{|c|c|c|c|c|c|c|c|}
\hline \multirow[t]{3}{*}{ Microorganism } & \multicolumn{7}{|c|}{ Zone of inhibition (mm) } \\
\hline & \multicolumn{2}{|l|}{ S. oleosa } & \multicolumn{2}{|c|}{ C. carandas } & \multicolumn{2}{|c|}{ S. koetjape } & \multirow{2}{*}{$\begin{array}{l}\text { Gentamicin } \\
\text { FJS }\end{array}$} \\
\hline & FJS & FDS & FJS & FDS & FJS & FDS & \\
\hline P. aeruginosa MDR1 & $20.0 \pm 1.5$ & - & $22.0 \pm 3.6$ & $17.0 \pm 1.2$ & $13.0 \pm 1.0$ & - & $11.0 \pm 1.0$ \\
\hline P. aeruginosa MDR2 & $22.0 \pm 1.0$ & $21.0 \pm 0.9$ & $23.0 \pm 1.0$ & $15.0 \pm 1.2$ & $15.0 \pm 1.5$ & $10.0 \pm 4.9$ & $10.0 \pm 0.6$ \\
\hline K. pneumoniae ESBL & $15.0 \pm 2.6$ & - & $13.0 \pm 1.0$ & $7.7 \pm 0.5$ & - & - & $12.0 \pm 0.6$ \\
\hline E. coli $\mathrm{P} 174 \mathrm{ESBL}$ & $21.0 \pm 1.5$ & - & $19.0 \pm 1.0$ & $15.0 \pm 0.5$ & $14.0 \pm 1.0$ & - & $9.3 \pm 0.6$ \\
\hline A. baumannii MDR1 & $20.0 \pm 2.1$ & - & $21.0 \pm 1.5$ & $9.7 \pm 0.9$ & $15.0 \pm 1.5$ & - & $8.7 \pm 0.6$ \\
\hline A. baumannii MDR2 & $20.0 \pm 2.0$ & - & $21.0 \pm 1.2$ & $11.0 \pm 1.2$ & $15.0 \pm 1.0$ & - & $11.0 \pm 0.6$ \\
\hline E. faecalis & $18.0 \pm 1.0$ & - & $17.0 \pm 1.5$ & $12.0 \pm 0.5$ & $8.3 \pm 0.6$ & - & $15.0 \pm 0.6$ \\
\hline S. aureus MRSA1 & $20.0 \pm 2.5$ & $7.3 \pm 0.5$ & $21.0 \pm 1.5$ & $10.0 \pm 0.5$ & $16.0 \pm 1.5$ & - & $10.0 \pm 1.0$ \\
\hline S. aureus MRSA2 & $21.0 \pm 1.5$ & - & $22.0 \pm 2.1$ & $13.0 \pm 0.8$ & $16.0 \pm 1.5$ & - & $10.0 \pm 1.0$ \\
\hline
\end{tabular}

Values are means inhibition zone $(\mathrm{mm}) \pm$ SD of three replicates. FJS: Fresh juice sample, FDS: Freeze drying sample, P. aeruginosa: Pseudomonas aeruginosa,

K. pneumoniae: Klebsiella pneumoniae, E. coli: Escherichia coli, A. baumannii: Acinetobacter baumannii, E. faecalis: Enterococcus faecalis, S. aureus: Staphylococcus aureus, MRSA: Methicillin-resistant Staphylococcus aureus, S. oleosa: Schleichera oleosa, C. carandas: Carissa carandas, and S. koetjape: Sandoricum koetjape

activity of against six organisms. The highest inhibition was found in S. aureus with an inhibition zone of $15 \mathrm{~mm}$ [23]. The presence of various phytochemical constituents in the extracts of $S$. oleosa influenced antioxidant activity. Leaf and seed extracts of S. koetjape was performed for the phytochemical constituents and antimicrobial activity on clinical isolates from patients [24]. The phytochemical showed the presence of tannins, phenols, flavonoids, alkaloids, saponins, steroids, and cardiac glycosides which involved in antioxidant and antimicrobial properties. The antibacterial susceptibility assay of $C$. carandas leaf extracts was done against the pathogenic microbes [25]. Fruits of $C$. carandas also had some inhibitory activity against selected bacterial cultures [26]

According to the components of FDS of S. oleosa and S. koetjape, especially the lyophilize process, it showed the moderate inhibitory effect against bacteria. This may be due to the chemical changes. The previous study demonstrated that the presence of polyphenols may have contributed to this result, as many molecules of this chemical group can appear antimicrobial activity [27]. In the investigation of the FDS of $C$. carandas demonstrated the antimicrobial activity against the 10 tested microorganisms, showed that $C$. carandas was the potential antibacterial agent. The process of freeze dryer on $C$. carandas may cause less effect on the active antibacterial compartments which could lead to study more in the future.

The previous study of many fruit extracts for antibacterial properties was revealed, for example, fruit extract of Avicennia officinalis [28], fruit of Averrhoa carambola Linn. and Ziziphus mauritiana Lam., [29] and the dried fruit of Embelia basaal [30] showed potent broad spectrum antibacterial properties. Fruits are important constituents in human and animal nutrition; however, storage of fruits has limitations, because they are perishable and deterioration by microbial and environmental factors that has a strong influence in shelf life. As a result, the stability of juice depends on the preparation, for example, lyophilize or freeze drying process becomes an important way to improve the value and storage of properties of fruit juices during the period of plenty and low costs of the seasonal fruits.

\section{CONCLUSION}

The results of this study reveal that the fresh juice extracts of S. oleosa have broad ranges of antibacterial activities and could be good potential sources for screening programs of bioactive natural products. The antioxidant activities demonstrated clearly that these extracts contain a number of antioxidant compounds which can effectively scavenge DPPH under in vitro condition. Its suggested that multiple mechanisms are involved. This study indicated that the extracts from S. oleosa, are the best sources of natural antimicrobial which might be useful in treating the diseases associated with pathogenic bacteria. Moreover, the freeze dry extraction process may be a cause of slightly decreasing of these antioxidant activities, but significantly decreasing in antimicrobial activity, which results of loss of phenolic compounds due to its heat sensitive property. This report may be useful for evaluation of pharmaceutical or treatment of infections depending on the extraction process. Further analysis should be considered analysis of vitamins, minerals and dietary fiber of the selected fruit with increase options for extraction shelf life which serve as value-added products to local fruit. Furthermore, the processing fruit in the form of freeze drying could be the alternative method for providing the antioxidant properties for developed natural products for well preserve and save space. This report may useful for evaluation these advantage properties for pharmaceutical purpose of the fruits in the future.

\section{REFERENCES}

1. Xi Y, Fan X, Zhao H, Li X, Cao J, Jiang W. Postharvest fruit quality and antioxidants of nectarine fruit as influenced by chlorogenic acid. Food Sci Technol 2017;75:537-44.

2. Ongphimai N, Lilitchan S, Aryusuk K, Bumrungpert A, Krisnangkura K. Phenolic acids content and antioxidant capacity of fruit extracts from Thailand. Chiang Mai J Sci 2013;40(4):636-42.

3. Crowe KM, Murray E. Deconstructing a fruit serving: Comparing the antioxidant density of select whole fruit and $100 \%$ fruit juices. J Acad Nutr Diet 2013;113(10):1354-8.

4. Lim HK, Tan CP, Karim R, Ariffin AA, Bakar J. Chemical composition and DSC thermal properties of two species of Hylocereus cacti seed oil: Hylocereus undatus and Hylocereus polyrhizus. Food Chem 2010;119:1326-31

5. Rinaldi M, Caligiani A, Borgese R, Palla G, Barbanti D, Massini R. The effect of fruit processing and enzymatic treatments on pomegranate juice composition, antioxidant activity and polyphenols content. LWT Food Sci Technol 2013;53:355-9.

6. Goveas SW, Abraham A. Evaluation of antimicrobial and antioxidant activity of stem and leaf extracts of Coscinium fenestratum. Asian $\mathrm{J}$ Pharm Clin Res 2013;6(3):218-21.

7. Nurmahani MM, Osman A, Hamid FA, Ghazali M, Dek MS. Antibacterial property of Hylocereus polyrhizus and Hylocereus undatus peel extracts. Int Food Res J 2012;19(1):59-66.

8. Meshram N, Ojha M, Singh A, Alexander A, Ajazuddin KJ, Sharma M. Significance and traditional medicinal properties of Schleichera oleosa. Asian J Pharm Res 2015;5(1):61-4.

9. Verma K, Shrivastava D, Kumar G. Antioxidant activity and dna damage inhibition in vitro by a methanolic extract of Carissa carandas (Apocynaceae) leaves. J Taibah Univ Sci 2015;9:34-40

10. Sarma A, Sarmah P, Kashyap D, Dutta S, Mahanta M. antioxidant activity and nutraceutical property of the fruits of an ethno-medicinal plant: Carissa carandas $\mathrm{L}$. found in brahmaputra valley agro-climatic condition. J Pharm Sci Res 2015;7(2):55-7.

11. Nassar Z, Aisha A, Majid AA. The pharmacological properties of terpenoids from Sandoricum koetjape. WebMedCentral 2010;1(12):1311

12. Vulić JJ, Velićanski AS, Četojević-Simin DD, Šaponjac VT, Djilas SM, Cveković DD, et al. Antioxidant, antiproliferative and antimicrobial 
activity of freeze-dried raspberry. Acta Period Technol 2014;45:99-116.

13. Vegara S, Mena P, Martí N, Saura D, Valero M. Approaches to understanding the contribution of anthocyanins to the antioxidant capacity of pasteurized pomegranate juices. Food Chem 2013;141:1630-6.

14. Powthong P, Thongmee A, Suntornthiticharoen P. Antioxidant and antimicrobial activities of endophytic fungi isolated from Sesbania grandiflora $($ L.) Int J Phytomed 2013;5:102-7.

15. Powthong P, Jantrapanukorn B, Thongmee A, Suntornthiticharoen P. Screening of antimicrobial activities of the endophytic fungi isolated from Sesbania grandiflora (L.) pers. J Agric Sci Technol 2013;15:1513-22.

16. Palanuvej C, Vipunngeun N. Fatty acid constituents of Schleichera oleosa (Lour.) oken. Seed oil. J Health Res 2008;22(4):203.

17. Ghosh P, Chakraborty P, Mandal A, Rasul MG, Chakraborty M, Saha A. Triterpenoids from Schleichera oleosa of darjeeling foothills and their antimicrobial activity. Indian J Pharm Sci 2011;73(2):231-3.

18. Fidrianny I, Rizki RK, Insanu M. In vitro antioxidant activities from various extracts of banana peels using ABTS, DPPH assays and correlation with phenolic, flavonoid, carotenoid content. Int J Pharm Pharm Sci 2014;6(8):299-303

19. Hardainiyan S, Nandy BC, Saxena R. Phytochemical investigation of fruit extract of Elaeocarpus ganitrus. Int J Pharm Pharm Sci 2015;7(6):415-8

20. Yan H, Kerr WL. Total phenolics content, anthocyanins, and dietary fiber content of apple pomace powders produced by vacuum-belt drying. J Sci Food Agric 2013;93(6):1499-504.

21. Sogi DS, Siddiq M, Greiby I, Dolan KD. Total phenolics, antioxidant activity, and functional properties of 'Tommy Atkins' mango peel and kernel as affected by drying methods. Food Chem 2013;141(3):2649-55.
22. Chellaram C, Parthasarathy V, Praveen MM, John AA, Anand TP, Priya G, et al. Analysis of phenolic content and antioxidant capacity of potato, Solanum tuberosum L from Tamil Nadu Region, India. APCBEE Proced 2014;8:105-8.

23. Khandekar U, Bobade A, Ghongade R. Evaluation of antioxidant activity, in-vitro antimicrobial activity and phytoconstituents of Schleichera oleosa (Lour.) oken. Int J Biol Pharm Res 2015;6(2):137-43.

24. Elijah AO, Onwuchekwa EC, Ekeleme UG. Phytochemical constituents and antimicrobial activity of Sandoricum koetjape leaf and seed extracts on clinical isolates from patients. Unique Res J Med Med Sci 2016;4(6):69-76

25. Agarwal T, Singh R, Shukla AD, Waris I. In vitro study of antibacterial activity of Carissa carandas leaf extracts. Asian J Plant Sci Res 2012;2(1):36-40

26. Patel PR, Rao TV. Effect of maturity indices of Carissa carandus L. Fruit on its antibacterial activity. Pharm Lett 2011;3(6):31-5.

27. Dias-Souza MV, dos Santos RM, de Siqueira EP, Ferreira-Marcal PH. Antibiofilm activity of cashew juice pulp against Staphylococcus aureus, high performance liquid chromatography/diode array detection and gas chromatography-mass spectrometry analyses, and interference on antimicrobial drugs. J Food Drug Anal 2016;1-8.

28. Sharief MD, Srinivasulu A, Veni PS, Rao VU. Quantification of phytochemicals and antibacterial activity of fruit extract of Avicennia officinalis. Asian J Pharm Clin Res 2014;7(2):127-30.

29. Das S. Antimicrobial and antioxidant activities of green and ripe fruits of Averrhoa carambola Linn. And Zizyphus mauritiana Lam. Asian J Pharm Clin Res 2012;5(3):102-5.

30. Barbade KD, Datar AG. Antibacterial activity, free radical scavenging potential, phytochemical investigation and in-vivo toxicity studies of medicinal plant Embelia basal (R. and S.) A. DC. Asian J Pharm Clin Res 2015;8(2):171-7. 